\title{
Breast Sparganosis Presenting with a Painless Breast Lump: Report of Two Cases
}

\author{
Moon Young Oh¹, Kyoung-Eun Kim¹, Min Jung Kim¹, Ajung Chu², Jong Yoon Lee², Jeong Hwan Park \\ Jongjin $\mathrm{Kim}^{4}$, Ki-Tae Hwang ${ }^{4, *}$ \\ 'Department of Surgery, Seoul National University Hospital, Seoul 03080, Korea; ${ }^{2}$ Department of Radiology, Seoul Metropolitan Government Seoul \\ National University Boramae Medical Center, Seoul 07061, Korea; ${ }^{3}$ Department of Pathology, Seoul Metropolitan Government Seoul National \\ University Boramae Medical Center, Seoul 07061, Korea; ${ }^{4}$ Department of Surgery, Seoul Metropolitan Government Seoul National University \\ Boramae Medical Center, Seoul 07061, Korea
}

\begin{abstract}
Sparganosis is a parasitic infestation caused by sparganum, a plerocercoid tapeworm larva of the genus Spirometra. Since the first case of human sparganosis reported in 1908, sparganosis has been a global disease, and is common in China, Japan, and Southeast Asian countries. Consumption of raw snakes, frogs, fish, or drinking contaminated beverages are sources of human infections. Human sparganosis usually manifests in subcutaneous fat in areas such as the abdomen, genitourinary tract, and limbs. Breast sparganosis cases are rare, representing less than $2 \%$ of total cases of human infections. Complete surgical extraction of the sparganum is the treatment of choice. Because of the rarity of the disease, clinical suspicion is vital to reach the diagnosis of breast sparganosis. Here we report 2 rare cases of breast sparganosis presenting with a painless breast lump, both treated with surgical excision and sparganum extraction.
\end{abstract}

Key words: Spirometra, breast, sparganosis, sparganum

\section{INTRODUCTION}

Sparganosis is a parasitic infestation caused by sparganum, a plerocercoid tapeworm larva of the genus Spirometra, including S. mansoni, S. ranarum, S. mansonoides, and S. erinacei. It was first discovered in China by Patrick Manson in 1882. The first human case was reported by Charles Wardell Stiles from Florida in 1908 [1]. Sparganosis is a global disease. It is common in China, Japan, and Southeast Asian countries [2]. Sparganum's life cycle begins when eggs are excreted into feces of dogs and cats. These eggs will hatch to become coracidia. After they are ingested by water fleas, their first intermediate host, they can grow into procercoid. When the second intermediate host, including frogs, snakes, and fish, ingests the daphnia, the larva can penetrate intestinal barriers and enter the tissue. The procercoid develops into plerocercoid (sparganum) in the second intermediate host, and the cycle is completed the final

- Received 18 January 2019, revised 19 March 2019, accepted 20 March 2019.

*Corresponding author (kiterius@snu.ac.kr)

(c) 2019, Korean Society for Parasitology and Tropical Medicine

This is an Open Access article distributed under the terms of the Creative Commons Attribution Non-Commercial License (http://creativecommons.org/licenses/by-nc/4.0) which permits unrestricted non-commercial use, distribution, and reproduction in any medium, provided the original work is properly cited. (definitive) hosts ingests the infected second intermediate host. The larva enters in an intestinal tract of the final host and becomes an adult worm. Humans can only be second intermediate hosts and are infected by eating raw snakes, frogs, fish, or drinking contaminated beverages $[2,3]$. The most common infection site of human sparganosis is subcutaneous fat in areas such as the abdomen, genitourinary tract, and limbs. Breast sparganosis is a rare disease that accounts for less than $2 \%$ of total cases of human infections [4]. Clinical suspicion is paramount to reach the diagnosis of breast sparganosis as these cases are rare. Patient's history is crucial. Results of radiological and laboratory tests are also helpful. Imaging tests used for diagnosis include mammography and breast ultrasonography that can be easily performed. Mammography shows irregular soft tissue density without calcification. Ultrasonography shows a long, folded banded low-echo structure within the heterogenous mass [5-7]. Here we report 2 rare cases of breast sparganosis with related literature review.

\section{CASE RECORD}

\section{Case 1}

A 69-year-old woman presented with a painless mass of the 


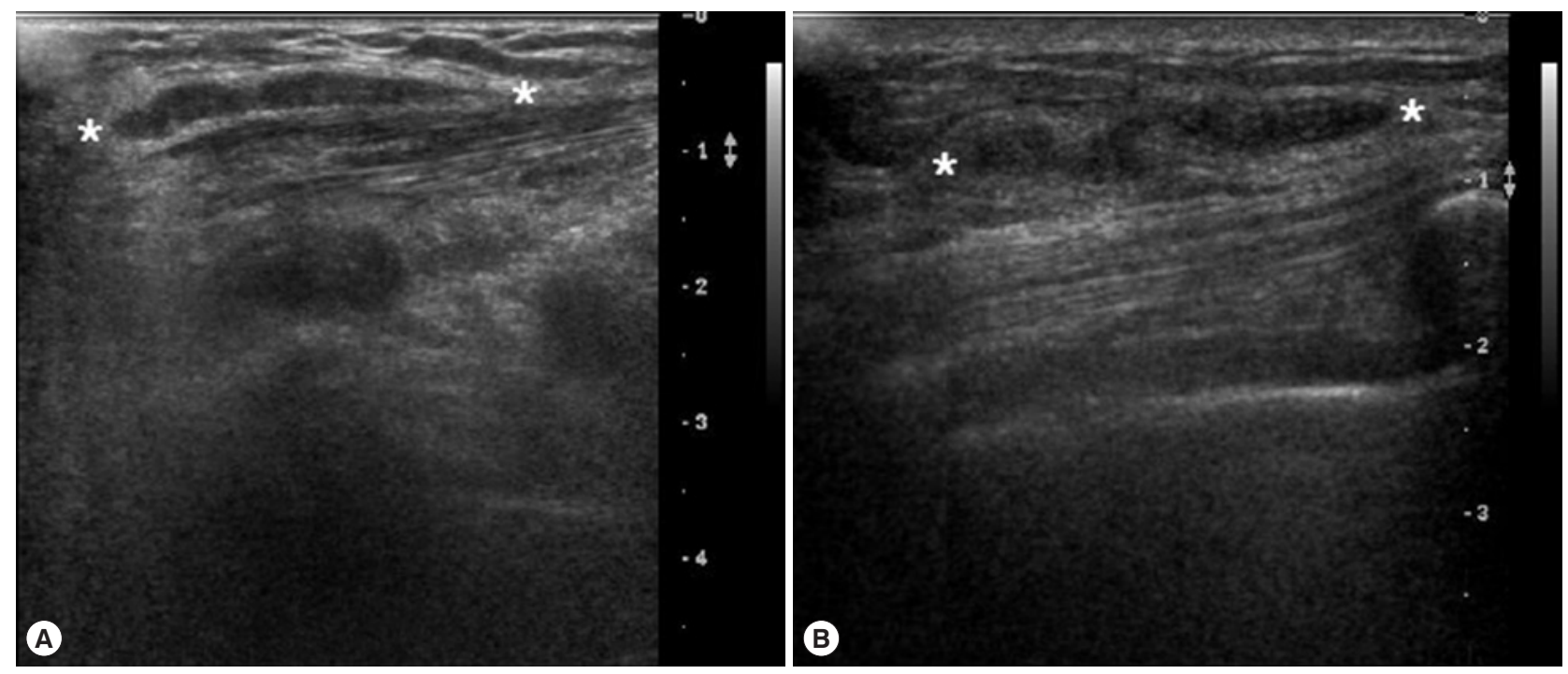

Fig. 1. Breast ultrasonographic findings. A long tubular low echoic structure in the subcutaneous fat layer of the infected breast in case 1 (A) and case 2 (B). *Indicates the long tubular low echoic structure in the infected breast.
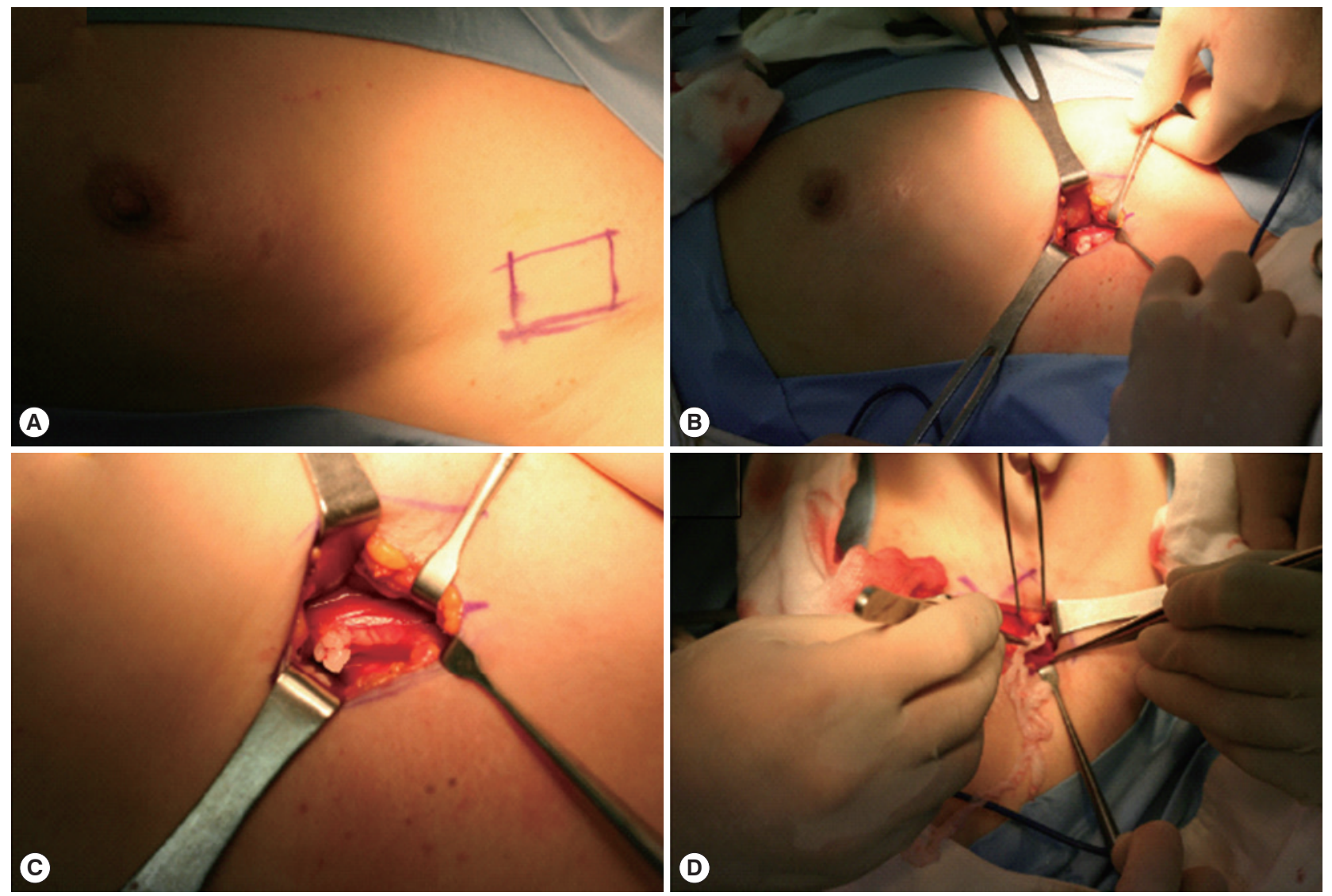

Fig. 2. Intraoperative findings of case 1. (A) A lesion is located between the left upper outer part of the breast and the axilla. (B) Skin incision is done over the main lesion and a worm is detected between the pectoralis muscles. (C) A close-up picture of the worm. (D) A worm is being extracted. 


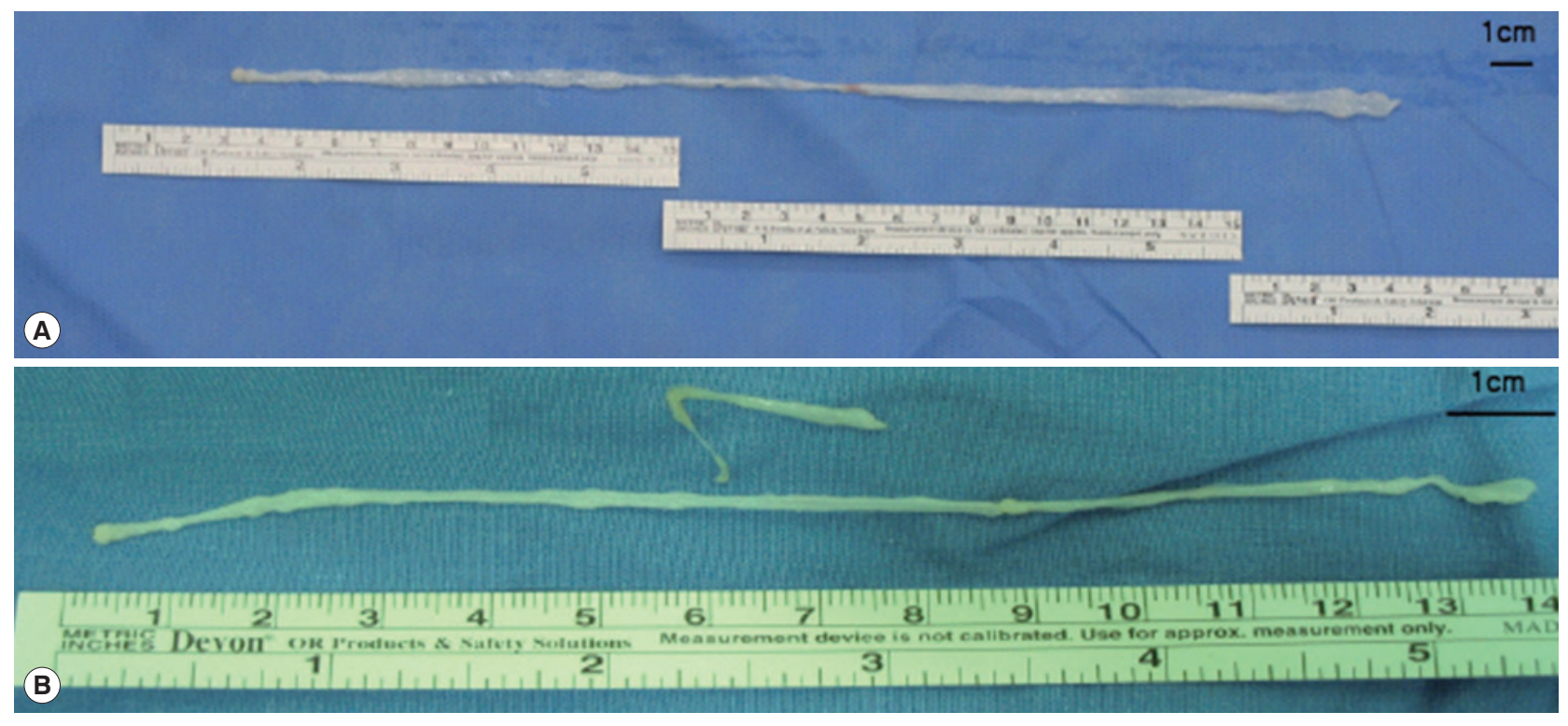

Fig. 3. Gross specimens. A ribbon-like whitish worm was extracted each from the infected breast in case 1 (A) and 2 (B). Size of sparganum was $30.0 \times 0.3 \mathrm{~cm}$ and $16.0 \times 0.2 \mathrm{~cm}$ in case 1 and 2 , respectively.

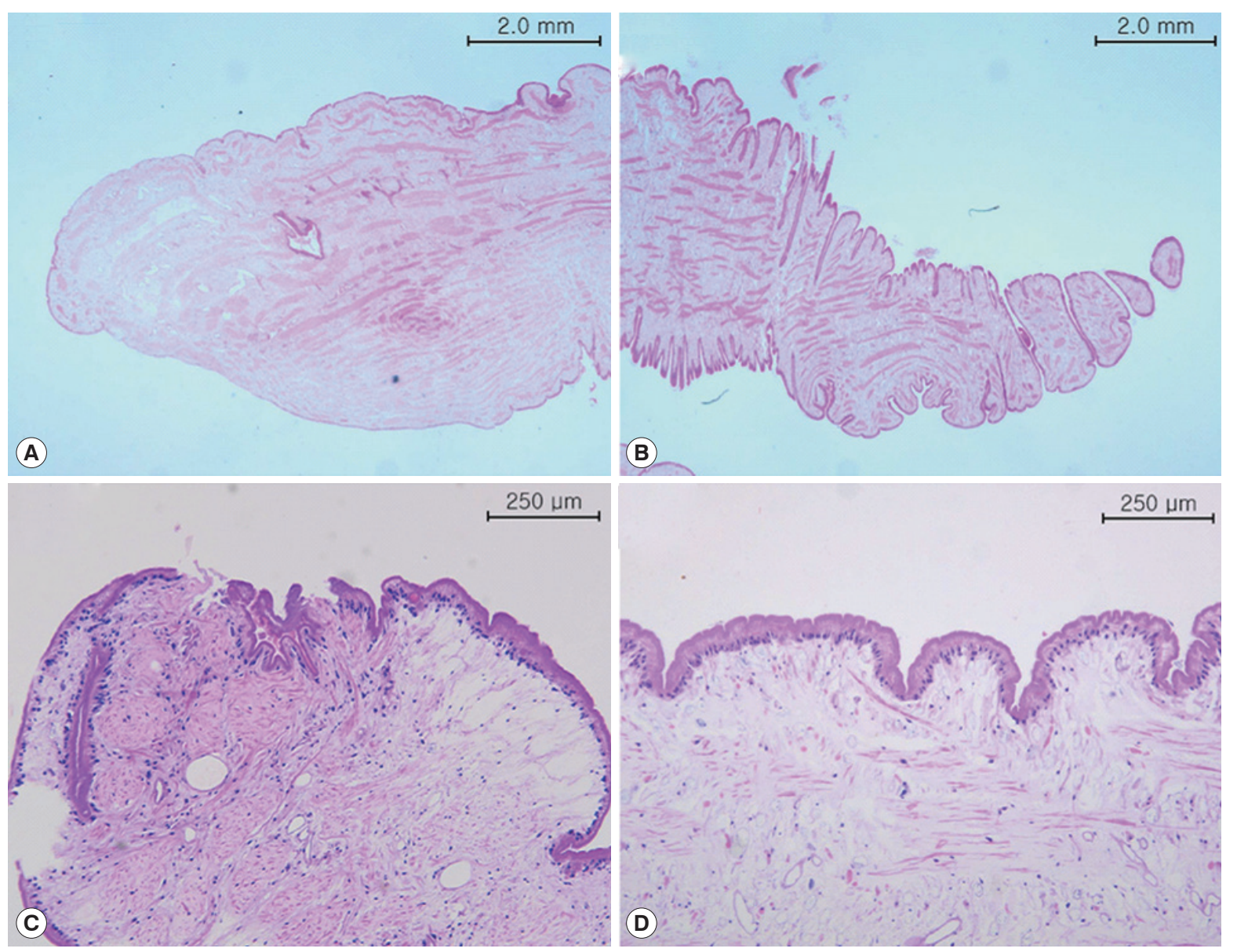

Fig. 4. Microscopic findings. The anterior (A) posterior end (B) of sparganum in case 1 (H\&E stain). The anterior end (C) and body part (D) of sparganum in case 2 (H\&E stain $\times 100)$. 
left upper breast. The mass was palpable 3 weeks prior to presentation. The patient was first treated with acupuncture at another hospital. The patient had no underlying disease other than hypertension. She denied any family history. The patient also denied eating raw foods, including snakes and frogs, although she did enjoy eating Korean-style raw beef from time to time. The patient did not complain of pain or pruritus at the lesion site. Although the mass had been palpable for 3 weeks, it was not palpable 3 days prior to visit. On physical examination, there was no mass found on the breast. There were no specific findings on other parts of the body. Laboratory findings were as follows: hemoglobin of $13.1 \mathrm{~g} / \mathrm{dl}$ and white

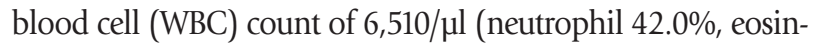
ophil 5.0\%). Chest X-ray and electrocardiogram (ECG) findings were normal. Mammography showed only benign calcifications of the left breast. On breast ultrasonography, a tubular hypoechoic lesion was seen at the subcutaneous fat layer in the left upper outer quadrant of the breast. The tip was connected to the back of the pectoral major muscle (Fig. 1A). A parasitic infection of the breast was suspected, and excisional biopsy was performed. During the operation, a worm surrounded by membranes located between the pectoral muscles was visually identified and removed (Fig. 2). The color of the identified worm was white. Its shape was thin and long, with a length of $30 \mathrm{~cm}$ and an outer diameter of $0.3 \mathrm{~cm}$ (Fig. 3A). Pathological examination demonstrated characteristic features of sparganum, including tegumental brush border, bundles of longitudinal muscle fibers, and lack of a gastrointestinal tract (Fig. 4A, B). Her postoperative course was uneventful. Followup was performed for one month postoperatively. No specific findings were observed.

\section{Case 2}

A 46-year-old woman presented with a painless mass of the right breast. The mass was palpated 4 months prior to presentation. The patient was followed up without any special treatment. She presented to the hospital because of recent increase in size of the lesion. The patient was taking medication for angina symptoms. She had no other underlying diseases. There were no specific findings in her family history. She denied of any recent consumption of raw food. The patient did not complain of pain, pruritus, or other symptoms in association with the lesion. She did not complain of any particular symptoms in other parts of the body. On physical examination, a $2 \mathrm{~cm}$ sized painless mass in the right upper part of the breast had grown in size compared to her last visit. The mass was hard and well-defined. There was no visual skin depression or color change. Laboratory findings were as follows: hemoglobin of $11.7 \mathrm{~g} / \mathrm{dl}$ and WBC count of 5,230/pl (neutrophil 35.5\%, eosinophil 3.1\%). Chest X-ray and ECG findings were normal. On mammography, there was no specific finding other than an asymmetric hyperdense lesion on the right upper side of the breast. A hypoechoic tubular lesion was observed on breast ultrasonography (Fig. 1B). A parasitic infection of the breast was suspected, and excisional biopsy was performed. During the operation, white circular figures filling the inside of the mass were visible. Length and outer diameter were $16 \mathrm{~cm}$ and $0.2 \mathrm{~cm}$, respectively (Fig. 3B). Pathologic examination of the tissue surrounding the worm showed chronic granulomatous inflammation accompanied by necrosis. The worm was confirmed as sparganum and microscopic findings showed characteristic thick eosinophilic tegument, subtegumental calcareous bodies, and longitudinal strips of muscles (Fig. 4C, D). Follow-up was done for up to 13 months postoperatively. No specific findings were observed during follow-up.

\section{DISCUSSION}

Sparganosis is a parasitic infestation caused by sparganum, a larva of genus Spirometra belonging to family Diphyllobothriidae [8]. The first case of sparganosis in Korea was reported by Uemura in 1917 and was rarely reported afterwards [4,9]. In most sparganosis cases reported in Korea, patients had histories of eating raw snakes and frogs [10]. However, in breast sparganosis cases reported so far, unlike those with sparganosis of other parts of the body, breast sparganosis patients do not have clear path of infection because they deny the history of eating raw snakes or frogs [11]. Likewise, in both cases reported here, patients do not have a history of ingesting snakes, frogs, or contaminated beverages, and thus, the pathway of this infection is uncertain. Therefore, detailed history taking is crucial, especially when the route of transmission is unclear.

Sparganum enters the human body and penetrates the wall of the digestive tract, making its way mainly to subcutaneous tissue and skeletal muscle. It can move to anywhere in the body and infect parts of the abdomen, bladder, epididymis, nervous system, eyeballs, and lungs [12-14]. When invading human tissues, larvae induces inflammatory edema within the stroma. When the larva dies, it causes strong local inflammation and necrosis [15]. Associated symptoms vary depending 
on the site of infection. In subcutaneous sparganosis, the parasite typically causes a painful inflammatory reaction in the tissues, and hence, patients complain of tenderness, dull pain, edema, or pruritus [1]. Sparganosis in the breast is a rare disease. Sometimes it presents as a painless breast mass. When the larva moves inside, the mass may appear to move [16]. In both cases described here, the patients present with breast masses without other symptoms such as pain or pruritus.

If breast sparganosis is suspected through review of patient's medical history and physical examination, mammography and breast ultrasonography can be used to help diagnose it. On mammography, the mass appears as an irregular soft tissue-density mass with clear margins without calcification. On ultrasonography, the sparganum body is observed as a hypoechoic structure inside a heterogeneous hyperechoic mass. The empty tract has a hypoechoic structure of similar shape. Granulation tissue around these structures is observed as a hyperechoic mass [5]. In the reported cases here, mammography alone is not enough to suspect parasite infection. It is through the detection of a tubular structure by breast ultrasonography that we were able to clinically suspect parasitic infection. Enzyme-linked immunosorbent assay (ELISA) test using antigens of plerocercoids can be used for the serodiagnosis of sparganosis. ELISA has a high sensitivity for detecting serum anti-sparganum antibodies in humans, but the main disadvantage is that cross-reactions with other parasitic diseases are possible [17]. Confirmation of sparganosis can be done surgically, and in both of the reported cases of this study, sparganum was easily confirmed with the naked eyes because its shape was preserved during surgery. Surgical treatment of sparganosis is a fundamental and effective treatment. Drugs such as praziquantel are sometimes administered alongside the surgical excision of the sparganum [18]. There are a few cases of recurrence after surgical removal of the sparganum [19]. Infection of sparganum can be prevented by avoiding eating raw snakes and frogs without drinking contaminated beverages.

Because sparganosis of the breast is often found as a painless breast mass, differential diagnosis of other tumors arising in the breast is essential. As sparganosis of the breast might be seen as a soft tissue nodule by radiologic exams, it is sometimes difficult to distinguish it from malignant tumor [20]. Thus, clinical suspicion is important to reach the diagnosis of breast sparganosis.

\section{CONFLICT OF INTEREST}

The authors declare that no actual or potential conflict of interest exists.

\section{REFERENCES}

1. Lescano AG, Zunt J. Other cestodes: sparganosis, coenurosis and Taenia crassiceps cysticercosis. Handb Clin Neurol 2013; 114: 335-345.

2. Beaver PC, Jung RC, Cupp EW, Craig CF. Clinical Parasitology. Philadelphia, USA. Lea \& Febiger. 1984, pp 499-504.

3. Lee BJ, Ahn SK, Kim SC, Lee SH. Clinical and histopathologic study of sparganosis. Korean J Dermatol 1992; 30: 168-174 (in Korean).

4. Min D. Cestode infections in Korea. Korean J Parasitol 1990; 28 : 123-144.

5. Kim HS, Cha ES, Kim HH, Yoo JY. Spectrum of sonographic findings in superficial breast masses. J Ultrasound Med 2005; 24: 663-680.

6. Kim YS, Hwang MS, Lee JK, Kim DS, Lee SK. US findings of breast sparganosis. J Korean Soc Med Ultrasound 2003; 22: 151156.

7. Chung SY, Park KS, Lee Y, Park CK. Breast sparganosis: mammographic and ultrasound features. J Clin Ultrasound 1995; 23: 447-451.

8. Kuchta R, Scholz T, Brabec J, Bray RA. Suppression of the tapeworm order Pseudophyllidea (Platyhelminthes: Eucestoda) and the proposal of two new orders, Bothriocephalidea and Diphyllobothriidea. Int J Parasitol 2008; 38: 49-55.

9. Koo M, Kim J, Kim JS, Lee JE, Nam SJ, Yang J. Cases and literature review of breast sparganosis. World J Surg 2011; 35: 573579.

10. Cho SY, Bae JH, Seo BS. Some aspects of human sparganosis in Korea. Korean J Parasitol 1975; 13: 60-77.

11. Park JH, Chai JW, Cho N, Paek NS, Guk SM, Shin EH, Chai JY. A surgically confirmed case of breast sparganosis showing characteristic mammography and ultrasonography findings. Korean J Parasitol 2006; 44: 151-156.

12. Suh Y, Choi WC, Choi YK. A case of ocular sparganosis in Korea. J Korean Ophthalmol Soc 2002; 43: 913-916 (in Korean).

13. Ahn JW, Chung CY, Bae WK. A case of sparganum mansoni in the thoracic and abdominal wall. J Korean Surg Soc 1967; 9: 419-421.

14. Anders K, Foley K, Stern WE, Brown WJ. Intracranial sparganosis: an uncommon infection: case report. J Neurosurg 1984; 60: 1282-1286.

15. Chang YK, Kim KC, Cho HJ. Sparganosis in the female breast. J Korean Surg Soc 2000; 58: 285-287 (in Korean).

16. Moon HG, Jung EJ, Park ST. Breast sparganosis presenting as a breast mass with vague migrating pain. J Am Coll Surg 2008; 207: 292. 
17. Liu LN, Zhang X, Jiang P, Liu RD, Zhou J, He RZ, Cui J, Wang ZQ. Serodiagnosis of sparganosis by ELISA using recombinant cysteine protease of Spirometra erinaceieuropaei spargana. Parasitol Res 2015; 114: 753-757.

18. Kwon OC, Kwak BS. Breast sparganosis: a case report. J Parasit Dis 2018; 42: 442-443.
19. Choi SJ, Park SH, Kim MJ, Jung M, Ko BH. Sparganosis of the breast and lower extremities: sonographic appearance. J Clin Ultrasound 2014; 42: 436-438.

20. Kim HY, Kang CH, Kim JH, Lee S, Park SY, Cho SW. Intramuscular and subcutaneous sparganosis: sonographic findings. J Clin Ultrasound 2008; 36: 570-572. 